\title{
TRANSITIONAL VORTICES IN WIDE-GAP SPHERICAL ANNULUS FLOW
}

\author{
E. O. IFIDON
}

Received 19 August 2004 and in revised form 6 May 2005

We develop a semianalytic formulation suitable for solving the Navier-Stokes equations governing the induced steady, axially symmetric motion of an incompressible viscous fluid confined in a wide gap between two differentially rotating concentric spheres. The method is valid for arbitrarily high Reynolds number and aids in the presentation of multiple steady-state flow patterns and their bifurcations. In the case of a rotating inner sphere and a stationary outer sphere, linear stability analysis is conducted to determine whether or not the computed solutions are stable. It is found that the solution transforms smoothly into an unstable solution beginning with asymmetric vortex pairs identified near the point of a symmetry-breaking bifurcation which occurs at Reynolds number 589. This solution transforms smoothly into an unstable asymmetric vortex solution as the Reynolds number increases. Flow modes whose branches have not been previously reported are found using this method. The origin of the flow modes obtained are discussed using bifurcation theory.

\section{Introduction}

Confined flows in rotating containers are known to exhibit multiple states, undergoing transitions from simple axisymmetric flows to complex asymmetric, irregular flows, with a multiplicity of structures including Taylor vortices. The classification of such multiple states and the transitions between them are of major importance in the understanding of geophysical phenomena and in the design of technological devices, and are therefore of fundamental interest for these systems [14]. Considerable information is available in the literature on the experimental classification of confined rotating flows [11], but analytic as well as numerical results are limited and incomplete especially for wide-gap spherical systems. There exists a need therefore, for a comprehensive semianalytical study, to map out the different flow regimes with a view to gaining better understanding of the phenomenon for wide-gap spherical annulus flow. Thus, this study focuses principally on the transitions between the different axisymmetric, steady, flow states in a confined spherical gap between a rotating inner sphere and a fixed outer spherical shell. The configuration to be studied involves the viscous incompressible flow in a wide gap between 


\section{Transitional vortices in wide-gap spherical annulus flow}

independently rotating concentric spheres, which permits variation of the geometric parameters and also allows co- or counter-rotation of the boundary spheres. We hope to obtain stability limits for wide-gap spherical shells that will show regions of stability of the steady states, including level curves corresponding to the various flow regimes. This study involves the use of a numerical method developed recently in a previous study to investigate steady-state axisymmetric confined swirling flows [5]. An extension of the numerical method is presented in this paper to include parameter continuation. The continuation technique enables us to follow branches of the steady-state solutions as a function of the Reynolds number. Linear stability analysis is conducted to determine whether or not the computed solutions are stable. It is found that the solution transforms smoothly into an unstable solution beginning with asymmetric vortex pairs identified near the point of a symmetry-breaking bifurcation which occurs at Reynolds number 589. Other bifurcation points are also identified which include a saddle-node bifurcation occurring at $\operatorname{Re} \approx 292$ which forms a pair of vortex branches one stable and the other unstable and two subcritical pitchfork bifurcation occurring at $\operatorname{Re} \approx 1100$. A stable Hopf bifurcation is detected at $\operatorname{Re} \approx 3000$, and although such bifurcations are known to exist in cylindrical Couette flow, they are unusual in purely axisymmetric spherical Couette flow.

\section{Governing equations}

We consider the motion of an isothermal incompressible viscous fluid contained in a spherical annulus between two rotating concentric spheres. The spheres are assumed to be rigid and rotate freely about a vertical axis through their centre, which is taken to be the origin of the coordinate system. The inner sphere and outer sphere are constrained to rotate with prescribed angular velocity $\omega_{1}$ and $\omega_{2}$, respectively. We start with the full incompressible Navier-Stokes equation with no body force [12];

$$
\frac{\partial \vec{q}}{\partial t}+\vec{q} \vec{\nabla} \cdot \vec{q}=-\vec{\nabla} p+\frac{1}{\operatorname{Re}} \nabla^{2} \vec{q}
$$

together with the incompressibility condition;

$$
\vec{\nabla} \cdot \vec{q}=0
$$

and the no-slip (rigid) boundary conditions on the spherical surfaces

$$
\begin{array}{ll}
\vec{q}=r \omega_{1} \sin \theta \hat{e}_{\varphi} & \text { at } r=r_{1}, \\
\vec{q}=r \omega_{2} \sin \theta \hat{e}_{\varphi} & \text { at } r=r_{2},
\end{array}
$$

where $\vec{q}=(u, v, w)$ is the velocity field, $p$ is the pressure, and Re is the Reynolds number defined by $\omega_{0} r_{2}^{2} / \nu$. In the two extreme cases, $\omega_{0}=\omega_{1}$ if the outer sphere is stationary and $\omega_{0}=\omega_{2}$ if the inner sphere is stationary. When both spheres rotate, the character of the flow is generally dominated by the motion of either the inner or outer sphere. The angular velocity of the dominant sphere is then taken as the characteristic angular velocity. The specific choice of $\omega_{0}$ is indicated for each example considered. The governing 
equations have been nondimensionalized with the characteristic velocity scale $\omega_{0} r_{2}$ and the characteristic length scale $r_{2}$. Since axisymmetric flows are considered, the stream function $\Psi$, vorticity function $\Phi$, and angular velocity function $\Omega$ can be introduced as follows:

$$
\begin{gathered}
u=\frac{1}{r^{2} \sin \theta} \frac{\partial}{\partial \theta} \Psi(r, \theta), \quad v=-\frac{1}{r \sin \theta} \frac{\partial}{\partial \theta} \Psi(r, \theta), \quad w=\frac{1}{r \sin \theta} \Omega(r, \theta), \\
(\vec{\nabla} \times \vec{q}) \cdot \hat{e}_{\varphi}=\frac{1}{r}\left[\frac{\partial}{\partial r}(r v)-\frac{\partial}{\partial \theta} u\right]=\frac{1}{r \sin \theta} \Phi(r, \theta) .
\end{gathered}
$$

The Navier-Stokes equations (2.1) and (2.2) are easily found to be

$$
\begin{gathered}
E^{2} \Omega=\frac{\operatorname{Re}}{r^{2} \sin \theta}\left[\Psi_{\theta} \Omega_{r}-\Psi_{r} \Omega_{\theta}\right], \\
E^{2} \Psi=\Phi, \\
E^{2} \Phi=\operatorname{Re}\left\{\frac{2 \Omega}{r^{3} \sin ^{2} \theta}\left[\Omega_{r} r \cos \theta-\Omega_{\theta} \sin \theta\right]+\frac{1}{r^{2} \sin \theta}\left[\Psi_{\theta} \Phi_{r}-\Psi_{r} \Phi_{\theta}\right]\right. \\
\left.+\frac{2 \Phi}{r^{3} \sin ^{2} \theta}\left[\Psi_{r} r \cos \theta-\Psi_{\theta} \sin \theta\right]\right\}=0,
\end{gathered}
$$

where

$$
E^{2}=\frac{\partial^{2}}{\partial r^{2}}+\frac{1}{r^{2}} \frac{\partial^{2}}{\partial \theta^{2}}-\frac{1}{r^{2}} \cot \theta \frac{\partial}{\partial \theta}
$$

In (2.5), (2.6), and (2.7), subscripts denotes partial differentiation.

In view of the boundary conditions, we may assume the expansions

$$
\begin{gathered}
\Omega(\mu, x)=\sum_{n=1}^{\infty} I_{2 n}(\mu) W_{n}(x), \\
\Psi(\mu, x)=\sum_{n=1}^{\infty} I_{2 n+1}(\mu) \psi_{n}(x), \\
\Phi(\mu, x)=\sum_{n=1}^{\infty} I_{2 n+1}(\mu) Y_{n}(x),
\end{gathered}
$$

where $I_{n}(\mu)$ are the Gegenbauer functions of argument $\mu=\cos \theta$. These functions form a complete orthogonal set in the range $-1 \leq \mu \leq 1$. We have substituted $r=e^{-x}$. Two advantages of solving the original problem in this fashion instead of a direct numerical integration of the partial differential equations are that for a given physical system, the computer time necessary to generate a solution in terms of the Gegenbauer polynomial expansion is less than that required to integrate the original partial differential equations. Also, the results in terms of numerical values for the radial dependence and the Gegenbauer polynomial representation to $\theta$ dependence are more convenient for stability 
2916 Transitional vortices in wide-gap spherical annulus flow

considerations than a set of values of $\Psi$ and $\Omega$ at various mesh points throughout the $r$ and $\theta$ range. Substituting (2.9), (2.10), and (2.11) in (2.5), (2.6), and (2.8) then making use of the orthogonality properties of the functions $I_{n}(\mu)$ give rise to infinite sets of ordinary differential equations. Substitution into (2.5) yields

$$
\frac{d^{2} W_{n}}{d x^{2}}+\frac{d W_{n}}{d x}-2 n(2 n-1) W_{n}=\sum_{i=1}^{\infty} A_{3}(x) \frac{d W_{i}}{d x}+\sum_{i=1}^{\infty} A_{4}(x) W_{i}
$$

where

$$
\begin{aligned}
& A_{3}(x)=-\operatorname{Re} \sum_{l=1}^{\infty} e^{x} \psi_{l}(x) d_{1}(2 n, 2 i, 2 l+1), \\
& A_{4}(x)=\operatorname{Re} \sum_{l=1}^{\infty} e^{x} \psi_{l}^{\prime}(x) d_{1}(2 n, 2 l+1,2 i) .
\end{aligned}
$$

By the use of similar procedures, (2.6) and (2.7) may be reduced to the respective sets of equations

$$
\begin{aligned}
& \frac{d^{2} \psi_{n}}{d x^{2}}+\frac{d \psi_{n}}{d x}-2 n(2 n+1) \psi_{n}=e^{-2 x} Y_{n}(x), \\
& \frac{d^{2} Y_{n}}{d x^{2}}+\frac{d Y_{n}}{d x}-2 n(2 n+1) Y_{n}=R_{n}(x),
\end{aligned}
$$

where $R_{n}(x)$ are nonlinear combinations of the various functions defined by

$$
\begin{aligned}
R_{n}(x)= & \sum_{i=1}^{\infty} A_{3}(x) \frac{d Y_{i}}{d x}+\sum_{i=1}^{\infty} A_{4}(x) Y_{i} \\
& +\operatorname{Re} \sum_{i=1}^{\infty} \sum_{l=1}^{\infty} e^{x}\left\{W_{i}(x) W_{l}^{\prime}(x) d_{2}(2 n+1,2 l, 2 i)\right. \\
& \left.\quad-2 W_{i}(x) W_{l}(x) d_{1}(2 n+1,2 i, 2 l)\right\} \\
A_{3}(x)= & -\operatorname{Re} \sum_{l=1}^{\infty} e^{x} \psi_{l}(x) d_{1}(2 n+1,2 i+1,2 l+1) \\
A_{4}(x)= & \operatorname{Re} \sum_{l=1}^{\infty} e^{x}\left[\psi_{l}^{\prime}(x)\left\{d_{1}(2 n+1,2 i+1,2 l+1)-d_{2}(2 n+1,2 l+1,2 i+1)\right\}\right. \\
& \left.\quad-2 \psi_{l}(x) d_{1}(2 n+1,2 i+1,2 l+1)\right]
\end{aligned}
$$

The quantities $d_{1}(n, m, k)$ and $d_{2}(n, m, k)$ which appear in (2.13), (2.16), (2.17), and (2.18) are integrals involving products of Gegenbauer functions and derived functions and are 
given by

$$
\begin{aligned}
d_{1}(n, m, k) & =\frac{n(n-1)(2 n-1)}{2} \int_{-1}^{1} \frac{I_{n}(\mu) I_{m}(\mu) P_{k-1}(\mu)}{1-\mu^{2}} d \mu \\
& =\sum_{i=0}^{n-2 i-2 \geq 0} \frac{(2 n-1)(2 n-4 i-3)}{2(2 m-1)} \int_{-1}^{1} P_{k-1}(\mu) P_{n-2 i-2}(\mu)\left\{P_{m-2}(\mu)-P_{m}(\mu)\right\} d \mu, \\
d_{2}(n, m, k) & =(n-1)(n)(2 n-1) \int_{-1}^{1} \frac{\mu I_{n}(\mu) I_{m}(\mu) I_{k}(\mu)}{\left(1-\mu^{2}\right)^{2}} d \mu \\
& =\frac{2 d_{1}(n, m, k)}{k}+\sum_{i=0}^{k-2 i-3 \geq 0} \frac{2(2 k-2 i-5)}{k(k-1)} d_{1}(n, m, k-2 i-2) .
\end{aligned}
$$

The boundary conditions corresponding to (2.12) are

$$
\begin{aligned}
& W_{n}\left(c_{1}\right)=2 h_{1} b_{1}^{2} \delta_{1 n}, \\
& W_{n}\left(c_{2}\right)=2 h_{2} b_{2}^{2} \delta_{1 n},
\end{aligned}
$$

where $c_{i}=-\log _{e} b_{i},\left(b_{i}=r_{i} / r_{2}\right)$ are the surfaces of the spheres, $h_{i}=\omega_{i} / \omega_{0}$, and $\delta_{n, 1}$ is the Kronecker delta function. The boundary conditions for (2.14) are

$$
\psi_{n}\left(c_{1}\right)=\psi_{n}\left(c_{2}\right)=\psi_{n}^{\prime}\left(c_{1}\right)=\psi_{n}^{\prime}\left(c_{2}\right)=0
$$

The functions $Y_{n}(x)$ are not known at the surface of the sphere and can be calculated as part of the solution. One way of doing this is to multiply (2.14) by $e^{-(2 n+1) x}$ and $e^{2 n x}$ in turn, then integrate both sides of the equation with respect to $x$ from $c_{1}$ to $c_{2}$. After some integration by part of the terms on the left-hand side and the use of conditions (2.21), we have

$$
\begin{aligned}
& \int_{c_{1}}^{c_{2}} e^{-(2 n+2) x} Y_{n}(x) d x=0, \\
& \int_{c_{1}}^{c_{2}} e^{-(2 n-1) x} Y_{n}(x) d x=0 .
\end{aligned}
$$

The use of (2.22) poses additional computational difficulties as their values need to be determined along with the other flow variables during the computational process. Conditions (2.22) act as an initial guess for the boundary conditions for $Y_{n}(x)$ and are to be improved upon iteratively during the computational process. In principle, any quadrature formulae may be used, but in practice, care need to be made about the choice of quadrature used as this may lead to inaccuracies and subsequent instability of the overall computational process. Thus, it would be computationally more advantageous, in order to circumvent the use of the conditions (2.22), to consider instead the fourth-order equation in $\psi_{n}(x)$. Thus, we set $\psi_{n}(x)=e^{-2 x} S_{n}(x)$ so that (2.14) becomes

$$
Y_{n}(x)=\frac{d^{2} S_{n}}{d x^{2}}+3 \frac{d S_{n}}{d x}+(2-2 n(2 n+1)) S_{n}
$$


2918 Transitional vortices in wide-gap spherical annulus flow

and (2.15) becomes

$$
\left[\frac{d^{2}}{d x^{2}}+\frac{d}{d x}-2 n(2 n+1)\right]\left[\frac{d^{2}}{d x^{2}}-3 \frac{d}{d x}+(2-2 n(2 n+1))\right] S_{n}(x)=R_{n}(x),
$$

where

$$
\begin{aligned}
R_{n}(x)= & \sum_{i=1}^{\infty} A_{3}(x)\left[\frac{d^{3}}{d x^{3}}-3 \frac{d^{2}}{d x^{2}}+(2-2 i(2 i+1)) \frac{d}{d x}\right] S_{i}(x) \\
& +\sum_{i=1}^{\infty} A_{4}(x)\left[\frac{d^{2}}{d x^{2}}-3 \frac{d}{d x}+(2-2 i(2 i+1))\right] S_{i}(x) \\
& +\operatorname{Re} \sum_{i=1}^{\infty} \sum_{l=1}^{\infty} e^{x}\left\{W_{i}(x) W_{l}^{\prime}(x) d_{2}(2 n+1,2 l, 2 i)\right. \\
& \left.-2 W_{i}(x) W_{l}(x) d_{1}(2 n+1,2 i, 2 l)\right\} .
\end{aligned}
$$

The boundary conditions imposed on $S_{n}(x)$ are

$$
S_{n}\left(c_{1}\right)=S_{n}\left(c_{2}\right)=S_{n}^{\prime}\left(c_{1}\right)=S_{n}^{\prime}\left(c_{2}\right)=0
$$

In the practical numerical integrations, the computations must be limited to the determination of a finite number of terms $n s$ in each of the series (2.9) to (2.11) by truncation of the series. We will determine later an appropriate choice for $n s$, for which terms with subscript greater than $n s$ will be set to zero.

\section{Computational method}

In this paper, we propose the use of a numerical technique which combines the basic ideas of homotopy analysis [7] and the collocation method in solving the systems (2.12) and (2.24). A family of formulae at different orders is given. At the lowest order, the current approach is the same as the traditional collocation method. Liao [6] and Liao and Chwang [8] successfully applied the homotopy analysis method to propose a general boundary element method for solving nonlinear problems. The basic ideas of homotopy are also applied to propose a noniterative numerical approach for two-dimensional viscous flow problems governed by the nonlinear Falkner-Skan equation and also in developing a kind of multigrid technique which requires less number of iterations and less CPU time than the traditional multigrid approach [9]. The basic idea is to construct a homotopy or mapping from a known or trivial solution (initial approximation) to the desired solution. This is achieved by first defining a homotopy or deformation in an embedding parameter $\lambda \in[0,1]$ as follows:

$$
H: \mathbb{R}^{n} \times[0,1] \longrightarrow \mathbb{R}^{n}
$$

such that

$$
H(x, 0)=G(x), \quad H(x, 1)=F(x),
$$


where $G: \mathbb{R}^{n} \rightarrow \mathbb{R}^{n}$ is a trivial or known solution. $H$ is assumed to be smooth. Typically, one chooses a homotopy such that

$$
H(x, \lambda):=(1-\lambda) G(x)+\lambda F(x)
$$

and attempts to trace an implicitly defined curve in the parametric set of this homotopy map from a starting point to a solution point. In order to ensure that this curve intersects the homotopy level $\lambda=1$ at finite points, it is sufficient to impose suitable boundary conditions which essentially prevent the curve from running to infinity before intersecting the homotopy level $\lambda=1$ or from returning back to the initial level. For a system of nonlinear pde,

$$
\Delta F(x)=0
$$

in a natural way. The idea is to start with a problem $\Delta_{0} G(x)=0$ with known or trivial solutions, then to continuously transform its solutions to those of the original problem (3.4). These solutions are the continuous functions corresponding to a family of differential operators $\Delta(x, \lambda) ; \lambda \in[0,1]$ with

$$
\Delta(x, 0)=\Delta_{0}, \quad \Delta(x, 1)=\Delta,
$$

given by

$$
\Delta(x, \lambda):=(1-\lambda) \Delta_{0}+\lambda \Delta,
$$

with

$$
\Delta(x, 0)=\Delta_{0}, \quad \Delta(x, 1)=\Delta .
$$

The corresponding homotopy equation is thus

$$
\Delta(x, \lambda) H(x, \lambda)=0
$$

or using (3.6),

$$
(1-\lambda) \Delta_{0} H(x, \lambda)+\lambda \Delta H(x, \lambda)=0 .
$$

We see from (3.9) that the process of $\lambda$ increasing from zero to one is just the process of $H$ going from $G(x)$, the initial guess, to $F(x)$, the desired solution. This process is called deformation in topology. Equation (3.9) is referred to as the zeroth-order deformation equation. Applying this concept of homotopy to the solutions of (2.12) and (2.24), for each $n$, we set

$$
H_{n}(x, \lambda)=W_{n}(x, \lambda)
$$

so that

$$
\begin{aligned}
& H_{n}(x, 0)=\Omega_{n}(x), \\
& H_{n}(x, \lambda)=W_{n}(x, \lambda) .
\end{aligned}
$$


2920 Transitional vortices in wide-gap spherical annulus flow

We start with the linear problem

$$
\Delta_{0} \Omega_{n} \equiv Q(x)\left[\frac{d^{2}}{d x^{2}}+\frac{d}{d x}-2 n(2 n-1)\right] \Omega_{n}(x)=0
$$

subject to the boundary conditions

$$
\begin{aligned}
& \Omega_{n}\left(c_{1}\right)=2 h_{1} b_{1}^{2} \delta_{1 n}, \\
& \Omega_{n}\left(c_{2}\right)=2 h_{2} b_{2}^{2} \delta_{1 n} .
\end{aligned}
$$

We then go on to solve the nonlinear equation

$$
\Delta W_{n} \equiv\left[\frac{d^{2}}{d x^{2}}+\frac{d}{d x}-2 n(2 n-1)\right] W_{n}(x)-\left[\sum_{i=1}^{\infty} A_{3}(x) \frac{d}{d x}+\sum_{i=1}^{\infty} A_{4}(x)\right] W_{i}(x)=0
$$

subject to (2.12). Therefore, (3.9) becomes for this problem

$$
(1-\lambda) \Delta_{0} W_{n}(x, \lambda)+\lambda \Delta W_{n}(x, \lambda)=0,
$$

with the corresponding boundary conditions

$$
\begin{aligned}
& W_{n}\left(c_{1}, \lambda\right)=2 h_{1} b_{1}^{2} \delta_{1 n} \\
& W_{n}\left(c_{2}, \lambda\right)=2 h_{2} b_{2}^{2} \delta_{1 n} .
\end{aligned}
$$

When $\lambda=0$, we see from (3.15) that

$$
W_{n}(x, 0)=\Omega_{n}(x)
$$

Also when $\lambda=1$, we see from (3.15) that

$$
W_{n}(x, 1)=W_{n}(x) .
$$

Thus, the process of $\lambda$ increasing from zero to one is just the process of $W_{n}$ varying from $\Omega_{n}(x)$ to $W_{n}(x)$. Obviously, (3.17) and (3.18) give a relationship between the initial guess $\Omega_{n}(x)$ and the solution $W_{n}(x)$. However this kind of relationship is indirect, a direct relationship between $\Omega_{n}(x)$ and $W_{n}(x)$ need to be obtained. Let us assume that the deformations $W_{n}(x, \lambda)$ are smooth enough about $\lambda=0$ so that all of the $m$ th-order deformation derivatives

$$
\Omega_{n}^{[m]}(x)=\left.\frac{\partial^{m} W_{n}(x, \lambda)}{\partial \lambda^{m}}\right|_{\lambda=0}, \quad m \geq 1,
$$

exist, then expanding $W_{n}(x, \lambda)$ in a Taylor series about $\lambda=0$ in the closed interval $[0,1]$, we have

$$
W_{n}(x, \lambda)=W_{n}(x, 0)+\sum_{m=1}^{\infty} \frac{\Omega_{n}^{[m]}(x)}{m !} \lambda^{m}
$$


Writing

$$
W_{m, n}(x)=\frac{\Omega_{n}^{[m]}(x)}{m !},
$$

we have from (3.17) that

$$
W_{n}(x, \lambda)=\Omega_{n}(x)+\sum_{m=1}^{\infty} W_{m, n}(x) \lambda^{m}
$$

Assuming the above Taylor series is convergent at $\lambda=1$, we have from (3.18) that

$$
W_{n}(x)=\Omega_{n}(x)+\sum_{m=1}^{\infty} W_{m, n}(x) .
$$

Expression (3.23) gives a direct relationship between the initial guess approximations $\Omega_{n}(x)$ and the required solution $W_{n}(x)$ through the unknown terms $W_{m, n}(x)$ whose defining equation can be obtained by differentiating the zeroth-order deformation equations (3.15) $m$ times with respect to $\lambda$ then setting $\lambda=0$, and finally dividing it by $m$ ! Thus

$$
\Delta_{0} W_{m, n}(x)=q_{m, n}(x), \quad m, n \geq 1,
$$

with the boundary conditions

$$
W_{m, n}\left(c_{1}\right)=W_{m, n}\left(c_{2}\right)=0, \quad m, n \geq 1 .
$$

The right-hand side term $q_{m, n}(x)$ is given by

$$
\begin{aligned}
q_{m, n}(x)= & \left(\chi_{m} \Delta_{0}-\left(\frac{d^{2}}{d x^{2}}+\frac{d}{d x}-2 n(2 n-1)\right)\right) W_{m-1, n}(x) \\
& +\left(\sum_{i=0}^{\infty} \sum_{k=0}^{m-1} A_{3, k} \frac{d}{d x} W_{m-1-k, i}(x)\right)+\sum_{i=1}^{\infty} \sum_{k=0}^{m-1} A_{4, k} W_{m-1-k, i}(x)
\end{aligned}
$$

and the coefficients $\chi_{m}, A_{3, l}(x)$, and $A_{4, l}(x)$ are defined by

$$
\begin{gathered}
\chi_{m}= \begin{cases}0 & \text { when } m \leq 1, \\
1 & \text { when } m \geq 2,\end{cases} \\
A_{3, l}(x)=-\operatorname{Re} \sum_{j=1}^{\infty} e^{x} \psi_{l, j}(x) d_{1}(2 n, 2 i, 2 j+1), \\
A_{4, l}(x)=\operatorname{Re} \sum_{j=1}^{\infty} e^{-x}\left(\frac{d}{d x} \psi_{l, j}(x)-2 \psi_{l, j}(x)\right) d_{1}(2 n, 2 j+1,2 i) .
\end{gathered}
$$


2922 Transitional vortices in wide-gap spherical annulus flow

Equation (3.24) is called the $m$ th-order deformation equation. It should be emphasized that all of the above $m$ th-order deformation equations are linear differential equations. Thus, through this approach, one solves an infinite sequence of linear subproblems instead of the original nonlinear equations (3.14). The initial approximation can be obtained from (3.12) and (3.13) given the value of $Q(x)$. Applying this concept of homotopy to the solutions of (2.24), for each $n$, we set

$$
H_{n}(x, \lambda)=\Psi_{n}(x, \lambda)
$$

so that

$$
\begin{gathered}
H_{n}(x, 0)=\phi_{n}(x), \\
H(x, 1)=S_{n}(x) .
\end{gathered}
$$

Neglecting the nonlinear terms in (2.24) should give us the desired initial guess for (2.24). However, the last nonlinear term on the right-hand side of (2.24) provides the "driving force" for this equation, and so cannot be neglected. One can therefore start with the problem

$$
\begin{aligned}
& \Delta_{0}\left(\phi_{n}(x), \Omega_{n}(x)\right) \\
& \quad \equiv Z(x)\left\{\left[\frac{d^{2}}{d x^{2}}+\frac{d}{d x}-2 n(2 n+1)\right]\left[\frac{d^{2}}{d x^{2}}-3 \frac{d}{d x}+(2-2 n(2 n+1))\right] \phi_{n}(x)-f_{n}(x)\right\},
\end{aligned}
$$

where $Z(x)$ is an arbitrary nonzero real-valued function and

$$
f_{n}(x)=\operatorname{Re} \sum_{i=1}^{\infty} \sum_{l=1}^{\infty} e^{x}\left\{\Omega_{i}(x) \frac{d \Omega_{l}}{d x} d_{2}(2 n+1,2 l, 2 i)-2 \Omega_{i}(x) \Omega_{l}(x) d_{1}(2 n+1,2 i, 2 l)\right\}
$$

with boundary conditions

$$
\phi_{n}\left(c_{1}\right)=\phi_{n}\left(c_{2}\right)=\frac{d \phi_{n}}{d x}\left(c_{1}\right)=\frac{d \phi_{n}}{d x}\left(c_{2}\right)=0
$$

then go on to solve the nonlinear problem

$$
\begin{aligned}
& \Delta\left(S_{n}(x), W_{n}(x)\right) \\
& \quad \equiv\left[\frac{d^{2}}{d x^{2}}+\frac{d}{d x}-2 n(2 n+1)\right]\left[\frac{d^{2}}{d x^{2}}-3 \frac{d}{d x}+(2-2 n(2 n+1))\right] S_{n}(x)-R_{n}(x),
\end{aligned}
$$


where

$$
\begin{aligned}
R_{n}(x)= & \sum_{i=1}^{\infty} A_{3}(x)\left[\frac{d^{3}}{d x^{3}}-3 \frac{d^{2}}{d x^{2}}+(2-2 i(2 i+1)) \frac{d}{d x}\right] S_{i}(x) \\
& +\sum_{i=1}^{\infty} A_{4}(x)\left[\frac{d^{2}}{d x^{2}}-3 \frac{d}{d x}+(2-2 i(2 i+1))\right] S_{i}(x) \\
& +\operatorname{Re} \sum_{i=1}^{\infty} \sum_{l=1}^{\infty} e^{x}\left\{W_{i}(x) \frac{d W_{l}}{d x} d_{2}(2 n+1,2 l, 2 i)-2 W_{i}(x) W_{l}(x) d_{1}(2 n+1,2 i, 2 l)\right\}
\end{aligned}
$$

subject to the boundary conditions (2.26). The choice of $Z(x)$ is very important as it completely determines $\Delta_{0}$. The zeroth-order deformation equation in an embedding parameter $\lambda$ is then

$$
(1-\lambda) \Delta_{0}\left(\Psi_{n}(x, \lambda), \Omega_{n}(x)\right)+\lambda \Delta\left(\Psi_{n}(x, \lambda), W_{n}(x, \lambda)\right)=0
$$

with the corresponding boundary conditions

$$
\Psi_{n}\left(c_{1}, \lambda\right)=\Psi_{n}\left(c_{2}, \lambda\right)=\left.\frac{d}{d x} \Psi_{n}(x, \lambda)\right|_{x=c_{1}}=\left.\frac{d}{d x} \Psi_{n}(x, \lambda)\right|_{x=c_{2}}=0
$$

When $\lambda=0$, we see from (3.35) that

$$
\Psi_{n}(x, 0)=\phi_{n}(x) .
$$

When $\lambda=1$, we see from (3.35) that

$$
\Psi_{n}(x, 1)=S_{n}(x)
$$

Thus, as $\lambda$ increases from zero to one, $\Psi_{n}(x, \lambda)$ increases from the initial approximation to the desired solution $S_{n}(x)$. Therefore, (3.37) and (3.38) give a relationship between the initial guess $\phi_{n}(x)$ and the solution $S_{n}(x)$. However, this kind of relationship is indirect. A direct relationship between $\phi_{n}(x)$ and $S_{n}(x)$ can be obtained as follows. Assume that the deformations $\Psi_{n}(x, \lambda)$ are smooth enough about $\lambda=0$ so that all of the $m$ th-order deformation derivatives

$$
\phi_{n}^{[m]}(x)=\left.\frac{\partial^{m} \Psi_{n}(x, \lambda)}{\partial \lambda^{m}}\right|_{\lambda=0}, \quad m \geq 1,
$$

exist, then by Taylor series, we have

$$
\Psi_{n}(x, \lambda)=\Psi_{n}(x, 0)+\sum_{m=1}^{\infty} \frac{\phi_{n}^{[m]}(x)}{m !} \lambda^{m} .
$$


2924 Transitional vortices in wide-gap spherical annulus flow

Writing

$$
\psi_{m, n}(x)=\frac{\phi_{n}^{[m]}(x)}{m !}
$$

we have from (3.37) that

$$
\Psi_{n}(x, \lambda)=\phi_{n}(x)+\sum_{m=1}^{\infty} \psi_{m, n}(x) \lambda^{m}
$$

Assuming the above Taylor series is convergent at $\lambda=1$, we have from (3.38) that

$$
S_{n}(x)=\phi_{n}(x)+\sum_{m=1}^{\infty} \psi_{m, n}(x)
$$

The above expression gives a direct relationship between the initial guess approximation $\phi_{n}(x)$ and the desired solution $S_{n}(x)$, through the unknown terms $\psi_{m, n}(x)$ whose governing equation can be obtained from (3.35) by differentiating the zeroth-order deformation equation (3.35) $m$ times with respect to $\lambda$, then setting $\lambda=0$ and finally dividing by $m$ !. Thus we have

$$
\Delta_{m, n} \psi_{m, n}(x)=h_{m, n}(x), \quad m \geq 1,
$$

where the linear differential operator $\Delta_{m, n}$ is given as

$$
\Delta_{m, n}=Z(x)\left[\frac{d^{2}}{d x^{2}}+\frac{d}{d x}-2 n(2 n+1)\right]\left[\frac{d^{2}}{d x^{2}}-3 \frac{d}{d x}+(2-2 n(2 n+1))\right]
$$

and the right-hand side term $h_{m, n}(x)$ is defined as

$$
\begin{aligned}
h_{m, n}(x)= & {\left[\chi_{m} \Delta_{m, n}-\left(\frac{d^{2}}{d x^{2}}+\frac{d}{d x}-2 n(2 n+1)\right)\right.} \\
& \left.\times\left(\frac{d^{2}}{d x^{2}}-3 \frac{d}{d x}+(2-2 n(2 n+1))\right)\right] \psi_{m-1, n}(x) \\
& +\sum_{i=1}^{\infty} \sum_{k=0}^{m-1} A_{3, k}\left(\frac{d^{3}}{d x^{3}}-3 \frac{d^{2}}{d x^{2}}+(2-2 i(2 i+1)) \frac{d}{d x}\right) \psi_{m-1-k, i}(x) \\
& +\sum_{i=1}^{\infty} \sum_{k=0}^{m-1} A_{4, k}\left(\frac{d^{2}}{d x^{2}}-3 \frac{d}{d x}+(2-2 i(2 i+1))\right) \psi_{m-1-k, i}(x) \\
& -\operatorname{Re} \sum_{i=1}^{\infty} \sum_{j=1}^{\infty} \sum_{k=0}^{m-1} e^{x}\left[\left(\frac{d}{d x} W_{k, j}\right) d_{2}(2 n+1,2 j, 2 i)\right. \\
& \left.-2 W_{k, l} d_{1}(2 n+1,2 i, 2 j)\right] W_{m-1-k, i}(x),
\end{aligned}
$$


where

$$
\begin{aligned}
& A_{3, l}(x)=-\operatorname{Re} \sum_{j=1}^{\infty} e^{-x} \psi_{l, j}(x) d_{1}(2 n+1,2 i+1,2 j+1) \\
& A_{4, l}(x)=\operatorname{Re} \sum_{j=1}^{\infty} e^{-x}[\left(-2 \psi_{l, j}(x)+\frac{d}{d x} \psi_{l, j}(x)\right) \\
& \times\left(d_{1}(2 n+1,2 i+1,2 j+1)-d_{2}(2 n+1,2 j+1,2 i+1)\right) \\
&\left.-2 \psi_{l, j}(x) d_{1}(2 n+1,2 i+1,2 j+1)\right] .
\end{aligned}
$$

With corresponding boundary condition

$$
\psi_{m, n}\left(c_{1}\right)=\psi_{m, n}\left(c_{2}\right)=\left.\frac{d}{d x} \psi_{m, n}(x)\right|_{x=c_{1}}=\left.\frac{d}{d x} \psi_{m, n}(x)\right|_{x=c_{2}}=0
$$

In the practical numerical integration of these equations, the computations must be limited to the determination of a finite number of terms in each of the series (2.9), (2.10), and (2.11) by truncation of the series. A truncation of order $n s$ is defined to be the process of setting to zero all terms with subscript greater than $n s$ in (2.9), (2.10), and (2.11) and likewise in (2.12) and (2.24) and solving the resulting set of $2 n s$ differential equations subject to the prescribed boundary conditions. The essence of homotopy is to transfer a nonlinear problem into an infinite number of linear subproblems and then to approximate the original nonlinear problem by the solutions of the first several linear subproblems. All of these linear subproblems are governed by the same linear operator, and therefore can be easily solved by numerical techniques. One can use the collocation method to solve the resulting boundary value problems to increase the numerical efficiency. The Matlab program bvp4c implements a collocation method for the solution of boundary value problems of the form

$$
y^{\prime}=f(x, y, p), \quad a \leq x \leq b
$$

Subject to a general nonlinear boundary condition,

$$
g(y(a), y(b), p)=0
$$

Here $p$ is a vector of unknown parameters.

Just about, any boundary value problem can be formulated for use with bvp4c. The first step is to write the ordinary differential equation (ODE) as a system of first-order ODEs. The process is described in [13]. In order to improve the efficiency of the solver, we combine the ideas of homotopy analysis and the collocation technique to develop a kind of collocation scheme for solving nonlinear problems. 
2926 Transitional vortices in wide-gap spherical annulus flow

Table 3.1. Number of iterations for the first- and second-order schemes.

\begin{tabular}{rcc}
\hline \multicolumn{1}{c}{$\operatorname{Re}$} & First-order scheme & Second-order scheme \\
\hline 263 & 34 & 17 \\
533 & 41 & 21 \\
1100 & 54 & 24 \\
\hline
\end{tabular}

Table 3.2. CPU time.

\begin{tabular}{rcc}
\hline Re & First-order scheme & Second-order scheme \\
\hline 263 & $45 \mathrm{~s}$ & $30 \mathrm{~s}$ \\
533 & $55 \mathrm{~s}$ & $42 \mathrm{~s}$ \\
1100 & $1 \mathrm{~min}, 30 \mathrm{~s}$ & $55 \mathrm{~s}$ \\
\hline
\end{tabular}

If we consider only the first $m$ terms in (3.23) and (3.43), we have the $m$ th-order approximations

$$
\begin{aligned}
& W_{n}(x) \approx \Omega_{n}(x)+\sum_{k=1}^{m} W_{k, n}(x), \\
& S_{n}(x) \approx \phi_{n}(x)+\sum_{k=1}^{m} \psi_{k, n}(x) .
\end{aligned}
$$

They provide us with the $m$ th-order iterative formulae

$$
\begin{aligned}
& \Omega_{n}^{i+1}(x) \longleftarrow \Omega_{n}^{i}(x)+\sum_{k=1}^{m} \Omega_{k, n}^{i}(x), \\
& \phi_{n}^{i+1}(x) \longleftarrow \phi_{n}^{i}(x)+\sum_{k=1}^{m} \phi_{k, n}^{i}(x),
\end{aligned}
$$

where $i=0,1,2, \ldots$ denote the iteration number.

If the first-order iterative formula is used, the current approach is just the same as the traditional collocation method. Our calculations show (Table 3.1) that the higher-order $(m=2)$ scheme seems more stable and converges faster than the direct application of the collocation method.

The solver requires the user to supply a guess for the solution as well as an initial mesh. The codes then adapt the mesh so as to obtain an accurate numerical solution with a modest number of mesh points.

From Table 3.2, the second-order scheme needs even less CPU time in all the cases than the first-order scheme. This is because the second-order scheme gives more accurate result than the first-order scheme, and therefore accelerates the iterations. The high-order iterative formula $m=2$ is used.

\section{Numerical results and discussion}

We consider the case of a rotating inner sphere and a stationary outer sphere, so that the Reynolds number is $\omega_{1} r_{2}^{2} / v$. For purposes of comparison with experimental results [10], 
the inner-to-outer radius ratio $b_{1}$ is taken to be 0.44 corresponding to a wide-gap spherical annulus flow. Wide-gap spherical annulus flow is suitable for simulating large scale astro- and geophysical flow and convection phenomena as they occur in the interior of the earth or other planets. Typical ratios of inner-to-outer radius are $b_{1}=0.34$ (innerto-outer earth core) and $b_{1}=0.54$ (outer-core-to-inner earth mantle). For gas planets, larger ratios are possible [1]. The calculations are carried out over a range of Re up to 4600 for a fixed value of $b_{1}$. For small Re, convergence is quite rapid and only a few terms are required to describe the properties of the solutions quite accurately. As the Reynolds number is increased, the number of terms required to give good accuracy also increases. Computations are carried out for several values of $n s$ for each value of Re and some judgment is made to the maximum value of $n s$ as required. As Re increases, more terms are required for convergence. A close examination of the computer output shows that in the exception of the endpoints, the first term used in the series representation of the stream functions, namely, $S_{1}(x)$, is positive definite and other terms, namely, $S_{n}(x), n>1$, can change sign. Also, we observe that for the cases considered, $S_{1}(x) \gg\left|S_{n s}(x)\right|$. This ensures the overall convergence of our computed solutions. A suitable choice of $Q(x), Z(x)$ need to be made to accelerate convergence of the series (3.23) and (3.43), respectively. For $\operatorname{Re} \leq 500$, we set $Q(x)=Z(x)=1$. If one were to set up this configuration in the laboratory, one of the most obvious measurements to be made would be the torque that must be exerted on the inner sphere to maintain its rotation. The torque is given by

$$
T^{*}=\int_{\phi=0}^{2 \pi} \int_{\theta=0}^{\pi} r^{3} \sin ^{2} \theta \tau_{\phi r}^{*} d \theta d \phi
$$

evaluated over the surface of the inner sphere, where $\tau_{\phi r}^{*}$ is the appropriate component of the stress tensor given by

$$
\tau_{\phi r}^{*}=\rho v\left[\frac{1}{r \sin \theta} \frac{\partial u^{*}}{\partial \theta}+r \frac{\partial}{\partial r}\left(\frac{\omega^{*}}{r}\right)\right]
$$

and $\rho$ is the density of the fluid [2]. If we substitute (4.2) in (4.1) with $\partial u^{*} / \partial \theta=0$ on the surface of the sphere, we obtain

$$
T^{*}=-\left.2 \pi \rho v a^{3} \omega_{0} \int_{0}^{\pi}\left(\frac{\partial \Omega}{\partial r}+2 \Omega\right)\right|_{r=r_{1}} \sin \theta d \theta .
$$

Then by substitution of the series (2.9) for $\Omega$, the integral in (4.3) can be evaluated in terms of $W_{1}(x)$ and its derivative at $c_{1}=-\log _{e} b_{1}$. If we introduce the dimensionless coeffiecient $M=-T^{*} /\left((1 / 2) \rho a^{5} \omega_{0}^{2}\right)$, then it is found that

$$
M=\frac{8 \pi}{3 \operatorname{Re}}\left[2 W_{1}\left(c_{1}\right)+W_{1}^{\prime}\left(c_{1}\right)\right] .
$$

The torque is thus dependent only on the derivative at $x=c_{1}$ of the first term of the series (2.9) and can be calculated quite accurately. The calculated value is a function of the order $n s$ of the truncation and converges very rapidly to a limit as $n s$ increases. This can provide a reasonable check of our numerical results and are also a convenient way of presenting 
Table 4.1. Comparison of the current results with experiments.

\begin{tabular}{ccc}
\hline $\mathrm{Re}$ & Experimental results $^{1}$ & Current study \\
\hline 480 & 0.460 & 0.458 \\
500 & 0.468 & 0.460 \\
530 & 0.480 & 0.473 \\
540 & 0.483 & 0.479 \\
\hline
\end{tabular}

${ }^{1}$ Values extracted from Figure 4.9 of Munson and Menguturk.

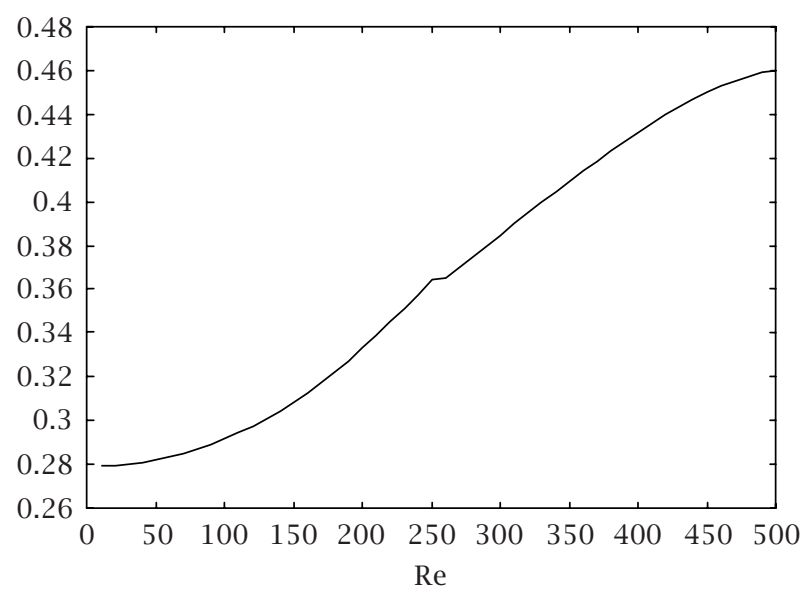

Figure 4.1. Torque distribution showing the onset of the first instability for $\operatorname{Re} \approx 250$.

our results. Table 3.1 provides a comparison of the current results with the experimental results of Munson and Menguturk (1975) see Table 4.1.

When the inner sphere rotates and the outer sphere remains stationary, plots of the torque distribution of different flow states are presented in Figure 4.1. The onset of the first instability occurs within the range $\mathrm{Re}<300$ which is in reasonable agreement with the experimental results of [10]. Figure 4.2 shows the second breakpoint in the torque distribution occurring at $\mathrm{Re} \approx 580$ which is also in reasonable good agreement with the experimentally measured value of 540 .

Matcont [3] is used to investigate the stability regions of the different flow modes. The Matlab-based toolbox is developed having in mind the detection of singularities via test functions and the processing of regular and singular points. A schematic representation of the bifurcation diagram generated is shown in Figure 4.3. The vertical axis represents the various flow states. Stable and unstable states are indicated with a + and - signs, respectively and are drawn with solid lines for the stable states and dashed lines for the unstable states. Linear stability analysis of the eigenvalues is used to determine the character of the flow modes. The solution is infinitesimally stable if all the eigenvalues have negative real parts. If at least one of the eigenvalues has a positive real part, then the solution is unstable. The second index of each label indicates the nature of the symmetry. S stands for symmetric, A stands for asymmetric, $\mathrm{O}$ stands for the basic flow mode without Taylor 


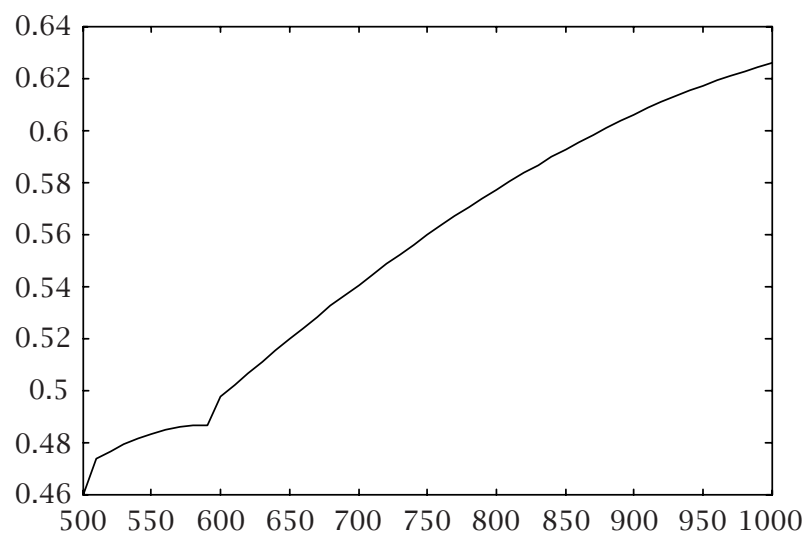

Figure 4.2. Torque distribution showing the second break in the Torque at $\operatorname{Re} \approx 580$.

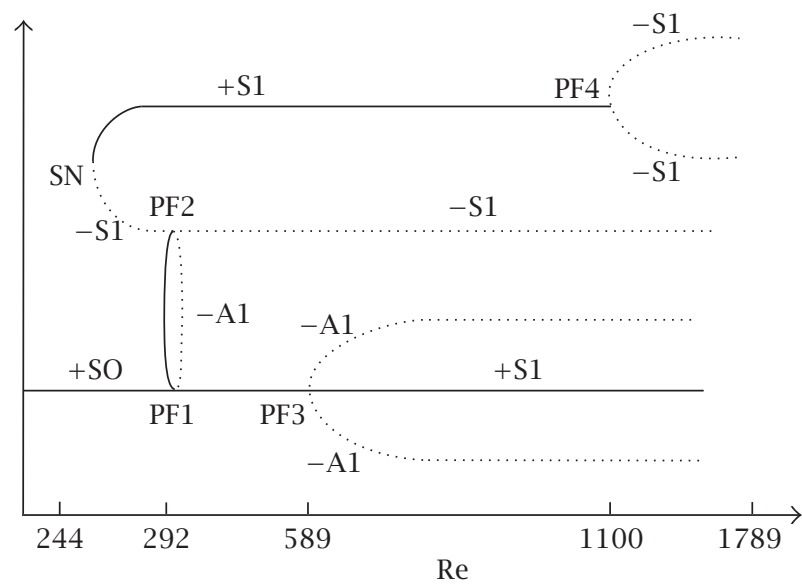

Figure 4.3. Schematic bifurcation diagram for $\operatorname{Re}<1800$.

vortices. Figure 4.3 suggests that the basic flow $(+\mathrm{SO})$ becomes an unstable vortex flow $(-S 1)$ via a subcritical pitchfork bifurcation. It is observed that there are no bifurcations between PF1 and PF3; this is consistent with the test function used in predicting and locating singularities in Matcont. Also the flow in the range $292<\operatorname{Re}<589$ is unstable with a single unstable eigenmode. The unstable mode undergoes another subcritical pitchfork bifurcation at $R e=589$. This leads to an unstable asymmetric vortex flow (-A1) whose asymmetry increases along its branch. As Re increases, the vortex increases in strength and size before finally settling to a stable vortex flow. This study identifies also the existence of a pair of unstable vortex branches as a result of a pitchfork bifurcation (PF4). One of the branches is characterized by a positive eigenvalue.

A plot is given of the variation of the radial velocity with radial distance at the equator for several values of Re. This is shown in Figure 4.4. We see that there is an increase of the 


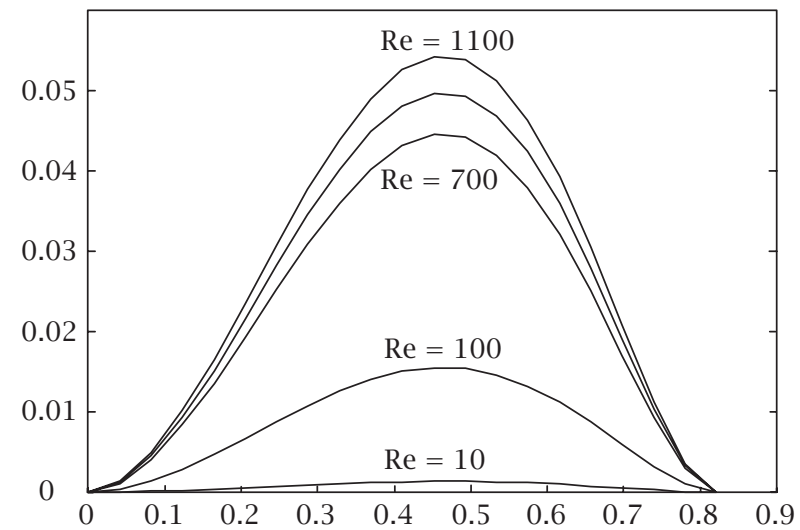

Figure 4.4. Variation of the radial velocity component with radial distance at the equator.

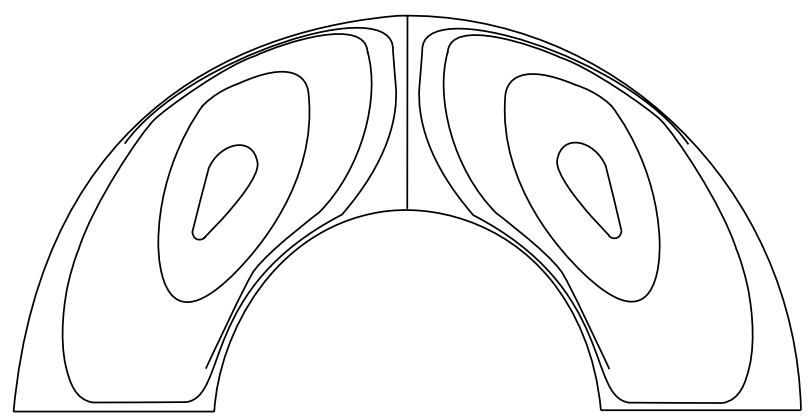

Figure 4.5. Contours of the meridional streamlines. Basic flow for $\operatorname{Re}=100$.

radial velocity component with Reynolds number at the equator. This increase suggests evidence of a jetting of the fluid from the equator of the inner sphere similar to a pumping effect, which causes the fluid to be thrown outward along the rotating inner sphere and drawn away from the stationary outer sphere. This results in a large-scale meridional flow whose direction is counter-clockwise in the northern hemisphere and clockwise in the southern hemisphere. This phenomenon can be seen in all flows with nonzero Reynolds number. Figures 4.5, 4.6, 4.7, 4.8, and 4.9 depict some of the flow modes predicted by this study. Figure 4.5 shows the basic flow at $R e=100$. It can be seen from the figure that the basic flow consists of a large-scale meridional circulation which is present on either side of the equator. The vortex-size distributions form closed curves which are almost circular.

Figure 4.7 is similar to Figure 4.6 in that it contains one pair of Taylor vortices near the equator; however its vortices are much strongly developed in terms of vortex strength and size than those of Figure 4.6. Figure 4.8 shows the asymmetric state at $\operatorname{Re}=600$. The stability analysis is carried out separately for each branch by solving a generalized eigenvalue problem. We only need to calculate those eigenvalues that are close to the imaginary axis since a change in them might change the stability of the solution. A Hopf bifurcation 


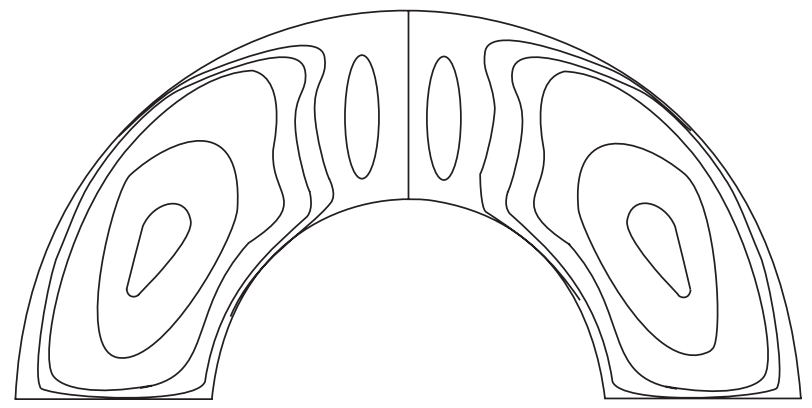

Figure 4.6. Contours of the meridional streamlines. Taylor vortex flow at $\mathrm{Re}=600$.

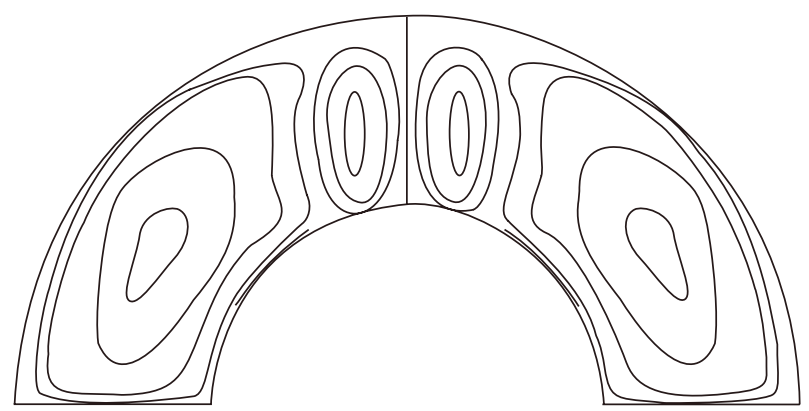

Figure 4.7. Contours of the meridional streamlines. Taylor vortex flow at $\mathrm{Re}=600$.

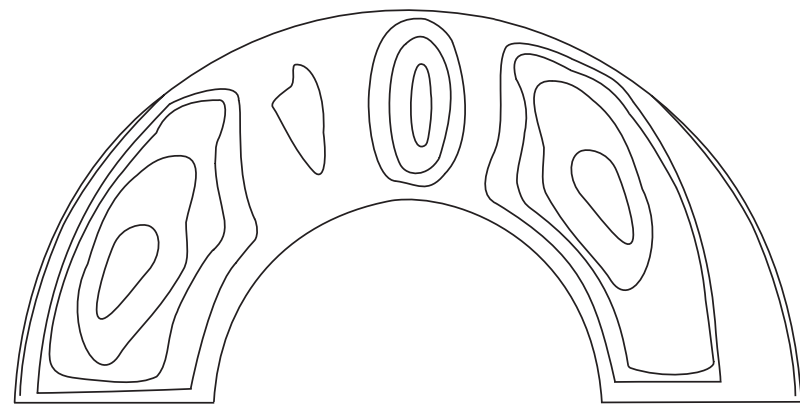

Figure 4.8. Asymmetric Taylor vortex at $\operatorname{Re}=600$.

point is detected if a complex pair of eigenvlaues crosses the imaginary axis. A stable supercritical Hopf bifurcation is detected for $\mathrm{Re} \approx 3000$ by the method described above; although such bifurcations are known to exist in cylindrical Couette flow, they are unusual in purely axisymmetric spherical Couette flow. No further bifurcations were observed using this method up to $\mathrm{Re}=4600$. 


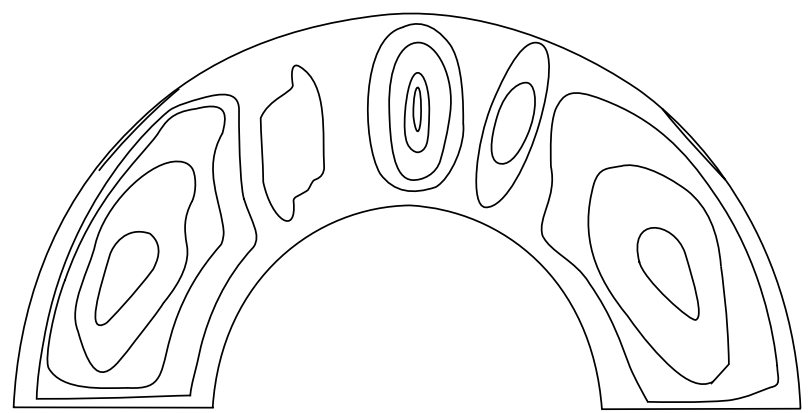

Figure 4.9. Asymmetric Taylor vortex at $\mathrm{Re}=3000$.

\section{References}

[1] C. Böhm, C. Egbers, and H. J. Rath, LDV- and PIV-measurements on thermal convection in spherical shells, Proc. 9th. International Symposium on Applications of Laser Techniques to Fluid Mechanics, Lisbon, July 1998.

[2] S. C. R. Dennis, S. N. Singh, and D. B. Ingham, The steady flow due to a rotating sphere at low and moderate Reynolds numbers, J. Fluid Mech. 101 (1980), 257-279.

[3] A. Dhooge, W. Govaerts, and Yu. A. Kuznetsov, MATCONT: a MATLAB package for numerical bifurcation analysis of ODEs, ACM Trans. Math. Software 29 (2003), no. 2, 141-164.

[4] R. Hollerbach, Time-dependent Taylor vortices in wide-gap spherical Couette flow, Phys. Rev. Lett. 81 (1998), no. 15, 3132-3135.

[5] E. O. Ifidon, Numerical studies of viscous incompressible flow between two rotating concentric spheres, J. Appl. Math. 2004 (2004), no. 2, 91-106.

[6] S. J. Liao, A direct boundary element approach for unsteady nonlinear heat transfer problems, Engineering Analysis with Boundary Elements 26 (2002), no. 1, 55-59.

[7] On the homotopy analysis method for nonlinear problems, Appl. Math. Comput. 147 (2004), no. 2, 499-513.

[8] S. J. Liao and A. T. Chwang, General boundary element method for unsteady non-linear heat transfer problems, International Journal of Numerical Heat Transfer B Fundamental 35 (1999), no. 2, 225-242.

[9] S. J. Liao and F. Mashayek, A multigrid approach for steady state laminar viscous flows, Internat. J. Numer. Methods Fluids 37 (2001), no. 1, 107-123.

[10] B. R. Munson and M. Menguturk, Viscous incompressible flow between concentric rotating spheres. III: Linear stability and experiments, J. Fluid Mech. 69 (1975), 705-719.

[11] J.-R. Schmidt, C. Egbers, and H. J. Rath, Experiments on the isothermal flow in wide spherical gaps, Proc. 12th International Couette Taylor Workshop, Illinois, September 2001.

[12] W. Sha and K. Nakabayashi, On the structure and formation of spiral Taylor-Gortler vortices in spherical Couette flow, J. Fluid Mech. 431 (2001), 323-345.

[13] The Mathworks, Using MATLAB, 24 Prime Park way, Natick, Massachusetts, 1996.

[14] R.-J. Yang and W.-J. Luo, Flow bifurcations in a thin gap between two rotating spheres, Theoret. Comput. Fluid Dynamics 16 (2002), no. 2, 115-131.

E. O. Ifidon: Department of Mathematics, The University of Benin, Ug bowo, PMB 1154, Benin City, Nigeria

E-mail address: ifidon@uniben.edu 


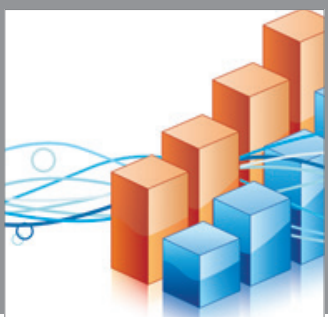

Advances in

Operations Research

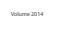

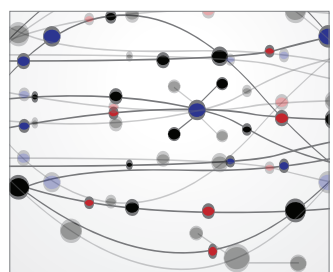

\section{The Scientific} World Journal
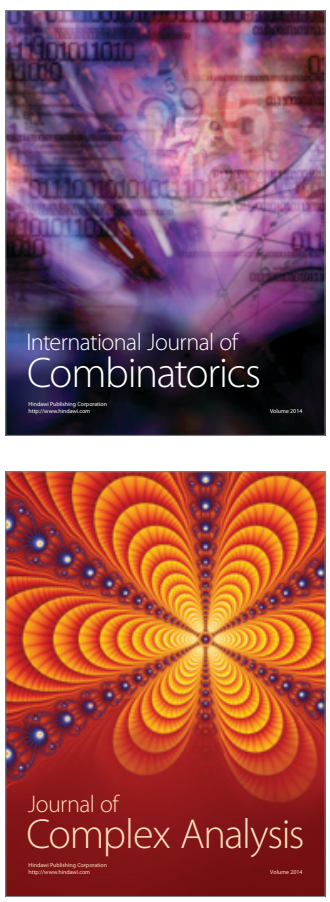

International Journal of

Mathematics and

Mathematical

Sciences
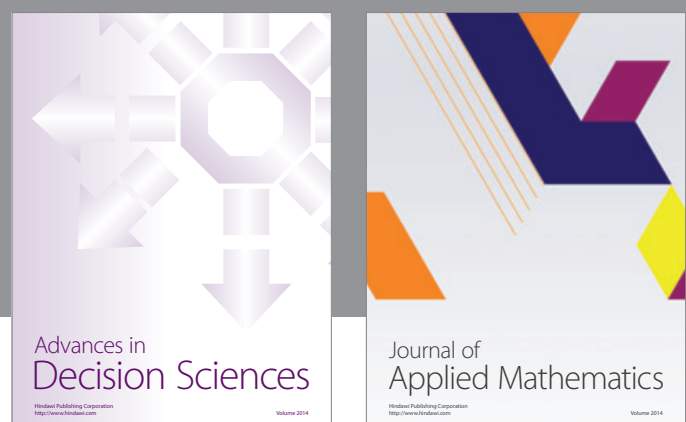

Journal of

Applied Mathematics
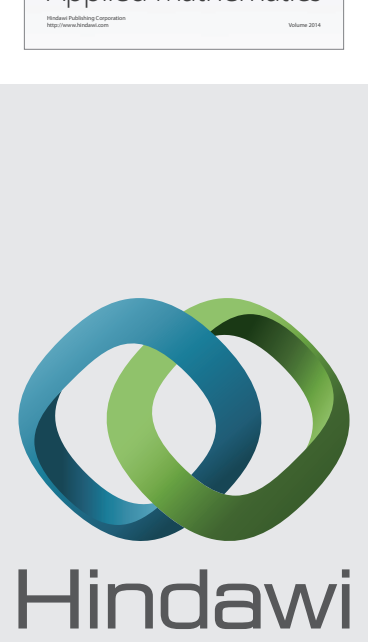

Submit your manuscripts at http://www.hindawi.com
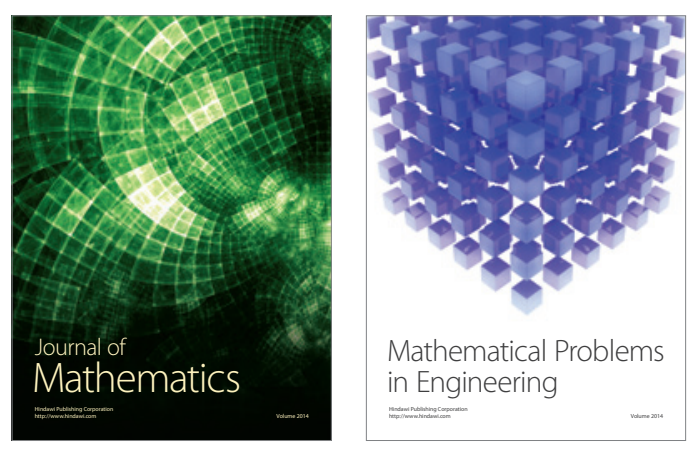

Mathematical Problems in Engineering
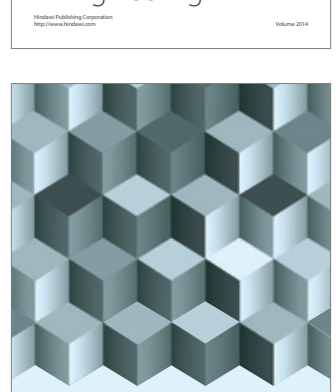

Journal of

Function Spaces
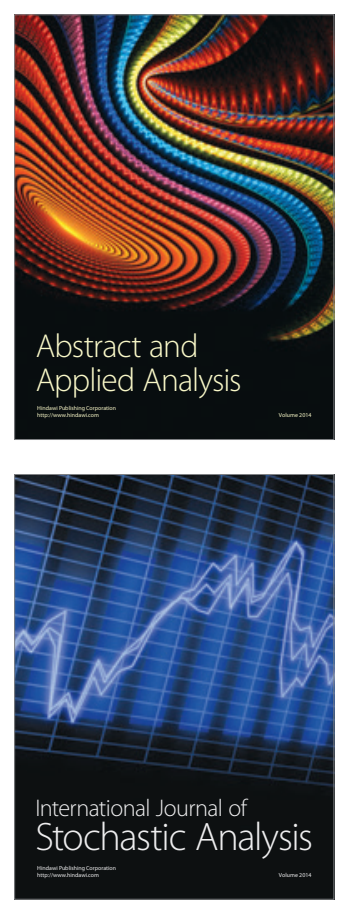

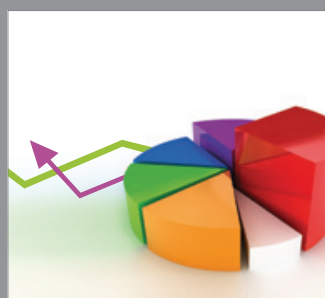

ournal of

Probability and Statistics

Promensencen
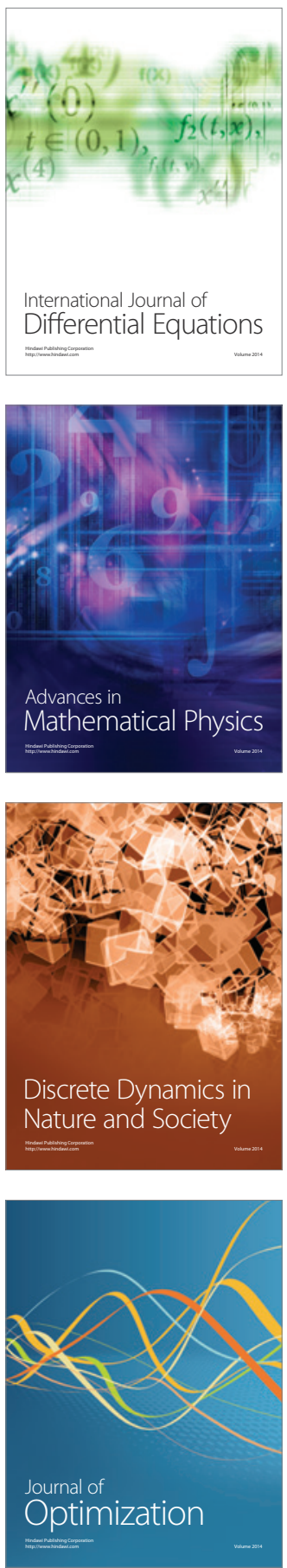